\title{
L'injure et la voix dans le théâtre de Shakespeare
}

\section{Nathalie Vienne-Guerrin}

\section{(2) OpenEdition}

\section{Journals}

Édition électronique

URL : http://journals.openedition.org/shakespeare/403

DOI : 10.4000/shakespeare.403

ISSN : 2271-6424

Éditeur

Société Française Shakespeare

Édition imprimée

Date de publication : 1 novembre 1999

Pagination : 193-208

ISBN : 2-84269-331-0

\section{Référence électronique}

Nathalie Vienne-Guerrin, «L'injure et la voix dans le théâtre de Shakespeare », Actes des congrès de la Société française Shakespeare [En ligne], 17| 1999, mis en ligne le 01 novembre 2007, consulté le 06 mai 2019. URL : http://journals.openedition.org/shakespeare/403 ; DOI : 10.4000/shakespeare.403 


\section{S H A K E S P E A R E \\ \& $\quad$ L $A \quad$ V O I X}

Société Française Shakespeare

Actes du Congrès de 1999

米米

Textes réunis et présentés par

Patricia DORVAL

publiés sous la direction de

Jean-Marie MAGUIN 


\section{L'I N JURE E T LA VOIX DA N S L E THÉ ÂT R DE SHAKESPEARE}

«Nothing my lord» ( $L R$, I.1.86). Il suffit de se rappeler le début de King Lear et notamment le mutisme de Cordelia qui refuse de donner à son père les caresses verbales qu'il lui réclame, pour constater qu'il est des injures sans voix, ou encore que rester sans voix peut être une injure, comme le rapelle Kate la mégère lorsqu'elle dit à propos de sa sœur «Her silence flouts me» (SHR, II.1.29).

Mais si l'injure peut passer par un geste, un regard, un silence, bref par d'autres voies / voix que celle des mots, il est cependant évident qu'injurier revient le plus souvent à «donner de la voix». Bien qu'elles soient souvent couchées sur le papier des anthologies de bons mots, les injures de théâtre passent en effet par le corps et notamment par le larynx de l'acteur qui produit ce que l'on appelle la voix. Par son oralité, l'insulte est inscrite dans le corps. Violence verbale, elle vient des tripes et agit sur les tripes («bowels») ${ }^{1}$. Elle vient de la gorge et va à la gorge. Dans le corpus shakespearien, environ un tiers des occurrences du terme «throat» (au singulier) ont un rapport direct avec l'insulte. L'ouverture de Richard II est de ce point de vue très emblématique, où Bolingbroke lance à Mowbray "With a foul traitor's name stuff I thy throat» (RII, I.1.44). Les pièces fourmillent d'allusions à cette dimension physique de l'injure qui va de pair avec une réification du signifiant et de la voix. Le mot est une flèche, un projectile ${ }^{2}$, or la voix est pour cette flèche un support indispensable. L'étymologie de termes tels que «vociférer» ou 
«provoquer» («pro-vocare») devrait suffire à nous persuader que le corps et la voix qu'il véhicule sont essentiels lorsqu'il s'agit d'injure. Dans son ouvrage ${ }^{3}$ consacré aux «gros mots», Pierre Guiraud parle de «l'expressivité vocale et corporelle» ${ }^{4}$ de l'injure et la relie au fait que «le juron et l'injure s'adressent directement - et physiquement - à un interlocuteur» ${ }^{5}$. L'injure, dit-il, «est un acte de parole, une parole qui est un acte par lequel le locuteur «affronte» physiquement son adversaire. Elle ne se contente pas de «dire quelque chose à propos de quelqu'un»; la voix qui conspue (c'est-à-dire «crache») fuse comme un crachat et s'accompagne souvent d'un crachat symbolique» ${ }^{6}$. Confondant le dire et le faire, l'injure est un domaine où la voix est reine et où le ton est roi, qui peut faire passer tout type d'énoncé à l'offensive.

Nous nous proposons ici de déterminer tout d'abord quelle(s) voix Shakespeare donne à l'injure en analysant les commentaires métalinguistiques que ses pièces nous offrent. Nous verrons ensuite dans quelle mesure la voix de l'injure shakespearienne est conditionnée par une syntaxe spécifique. Oscillant entre les deux pôles que sont le bruit et la musique, I'injure apparaîtra alors comme un espace dialectique où le dit et le dire se complètent et $s$ 'affrontent. Nous dégagerons trois modes essentiels de vocalisation de l'injure. Comme signifiant verbal et physique, l'injure semble produire un effacement du sens au profit de la signifiance. Mais nous verrons que Shakespeare sait aussi jouer sur l'effacement de la voix au profit du message, certaines formules injurieuses étant «gorgées» d'un sens qui devrait «neutraliser» la voix de l'injure. Enfin, nous examinerons comment le dramaturge exploite les décalages ironiques entre voix et message, entre le dit et le dire à des fins essentiellement comiques.

Il semble tout d'abord qu'avec l'injure, la voix absorbe le sens, ou que le sens disparaisse, comme englouti par la voix. Bref, l'injure, dans ses manifestations extrêmes, ne serait plus que voix. Selon Évelyne Largueche, les injures seraient «des cris déguisés en mots» ${ }^{7}$ ou encore «une parole où les mots sont davantage des gestes verbaux qu'un véritable langage» ${ }^{8}$. Évelyne Largueche compare l'insulte au cri du nourrisson qui constitue selon elle une manifestation vocale «en même temps qu'un effort déchirant pour ne pas étouffer» ${ }^{9}$. Les Élisabéthains, comme Puttenham, percevaient déjà l'injure comme une libération vocale. Ce dernier décrivait l'insulte, rappelons-le, comme «a great easement to the boiling stomach» ${ }^{10}$. Ainsi, l'injure serait, selon les termes d'Évelyne Largueche «une parole qui détourne en quelque sorte un instrument 
d'expression de la pensée par un envahissement de l'expression du corps» ${ }^{11}$. Shakespeare évoque à de nombreuses reprises cette réification du signifiant, qui fait des injures des poignards ${ }^{12}$, des dagues ${ }^{13}$, bref, des armes.

Évelyne Largueche explique alors que cette dimension corporelle de l'injure («non-spécifique» ${ }^{14}$ ) peut être qualifiée de sexuelle dans la mesure où les mots sont traités comme des objets, ce qui «redonne en quelque sorte corps à cette cavité qu'est la bouche, lui fait retrouver une importance sensorielle que l'activité de parole, en tant qu'instrument de transmission utilisant les mots pour leur sens, a tendance à faire oublier» ${ }^{15}$. On assiste à une «resexualisation» ${ }^{16}$ de la voix qui n'est plus support de mots et de sens mais qui devient le prolongement du corps. Évelyne Largueche note à propos des joutes d'injures ritualisées que «l'acte de parole représente alors symboliquement l'exhibition du penis, se taire c'est ne pas en avoir, ou être castré» ${ }^{17}$.

Si l'on relit l'absence de réaction de Romeo face à l'injure lancée par Tybalt («thou art a villain», $R O M$, III.1.60) à la lumière de ces considérations, l'épisode prend tout son sens. Romeo reste sans voix face à l'injure. Associée à des réflexions comme «Romeo [...] Without his roe» (ROM, II.4.37) ou à l'image d'un personnage que la beauté de Juliette a rendu «effeminé» («effeminate», ROM, III.1.116), cette inertie prend une signification sexuelle indéniable. Si la voix est une arme, rester sans voix revient à perdre son arme virile. A l'inverse, donner à la femme une voix de mégère, lui faire brandir des «poignards» verbaux ( $A D O$, II.1.246), c'est la doter d'une certaine virilité, virilité confirmée par certaines illustrations de l'époque qui représentent la femme en train de chevaucher son malheureux époux.

Cette lecture de la voix comme arme virile était déjà suggérée par Thomas Wright dans son ouvrage intitulé Passion of the Mind, où, soucieux d'établir des liens entre voix et passion, il narre l'anecdote suivante dans un chapitre intitulé «In Voyce»:

There came a man to Demosthenes desiring his helpe to defend his cause, and told him how one had beaten him: Demosthenes aunswered him againe, saying, I doe not beleeve this to be true thou tellest mee, for surely the other did never beat thee. The plaintife then thrusting out his voyce aloude, sayde; what, hath he not beaten me? Yes indeede quoth Demosthenes, I beleeve it now, 
for I heare the voice of a man that was beaten indeede: whereby we may see how hee coniectured, by the lowdnesse of his voyce, the iust indignation of his minde : For indeede, men in ire and wrath, shewe, by their pronunciation, the flame that lodgeth in their breasts. Wherefore Cato gave counsell, that soldiers, in the warre, should terrifie their enemies with vehement voyces and cries. ${ }^{18}$

Signe paralinguistique qui peut être lu et interprété, la voix donne lieu à une véritable codification qui était sans doute mise en œuvre par les acteurs élisabéthains ${ }^{19}$. Or les nombreux commentaires métalinguistiques présents dans les pièces de Shakespeare semblent montrer que l'injure comme fruit de la colère et excès de langage prête souvent aux excès de voix. «Be not so loud», dit Menenius à Volumnia qui est en train d'agresser vocalement ceux qui ont banni son fils Coriolan (Cor, IV.2.14); «Thou but offends thy lungs to speak so loud» (MV, IV.1.140) lance Shylock à Gratiano qui, fou de colère, I'injurie. Ces deux réactions expriment l'un des traits saillants de l'injure : son volume vocal. L'injure ne se dit pas, elle se crie, à tel point qu'il semble que la voix de l'injure disparaisse au profit du bruit. Le terme «rail» est l'un de ceux qui reviennent très souvent pour faire référence à l'acte d'insulter dans les pièces de Shakespeare. Or le terme «railler» français dont «rail» est dérivé, évoque par son étymologie différents types de bruits et de cris (notamment des bruits animaux). Celui ou celle qui insulte fait du bruit. C'est le cas bien sûr de la mégère Kate qui incarne en quelque sorte la voix de l'injure et dont on peut dire, en jouant sur l'équivalent allemand de l'expression «rough music», qu'elle produit une véritable «Kat[e]zen Music». C'est ce qu'indique le commentaire d'Hortensio lorsqu'il évoque les débordements verbaux de la mégère en les appelant «her loud alarums» (I.1.126-7). Pour Tranio, les invectives lancées par Kate constituent un vacarme («din», SHR, I.1.171-3). Petruchio parle du bruit de l'injure en ces termes:

Pet. Think you a little din can daunt mine ears?

Have I not in my time heard lions roar?

Have I not heard the sea puff'd up with winds, Rage like an angry boar chafed with sweat? Have I not heard great ordnance in the field, And heaven's artillery thunder in the skies? 
Have I not in a pitched battle heard

Loud 'larums, neighing steeds, and trumpets' clang?

And do you tell me of a woman's tongue,

That gives not half so great a blow to hear

As will a chestnut in a farmer's fire?

Tush, tush, fear boys with bugs !

(SHR, I.2.198-209)

En comparant les débordements verbaux de la mégère à des bruits de toutes sortes, Petruchio fait d'elle une présence avant tout sonore et inhumaine et du même coup lui fait perdre la voix, ce qui constitue une étape symbolique essentielle du dressage qu'il entreprend. La mégère n'a plus de voix, elle n'est plus que bruit.

Le texte shakespearien regorge d'images qui mettent l'accent sur la sonorité de l'injure. Les termes «roar», «thunder», «noise», et surtout «jars» 20 si souvent employés pour décrire les querelles renvoient au bruit de l'injure. Dans King John, les insultes sont appelées «ill-tuned repetitions» (JN, II.1.197). Basset dans 1 Henry VI fait de la voix de l'injure un aboiement lorsqu'il parle de «the envious barking of your saucy tongue» (III.8.33). Dans Coriolanus, Menenius décrit les insultes lancées par le héro éponyme comme des accents durs («rougher accents», III.3.54-5). Shakespeare traduit donc souvent l'essence sonore de l'injure et lui donne une voix dont on finit par se demander si elle en est bien une, tant elle est assimilée au cri et au bruit.

L'injurieur lance ses flèches verbales «à tue-tête» et cette tendance va de pair avec la syntaxe exclamative de l'injure qui se caractérise par une «sur-accentuation». L'absence de verbe, de noyau syntaxique et la prolifération de substantifs qui caractérisent l'injure conduisent en effet à une multiplication des syllabes accentuées. La récurrence du vocatif (étymologiquement lié à la voix et qui signifie «appeler») dans la syntaxe de l'injure peut entraîner une augmentation du volume vocal et constituer un tremplin pour la voix. Les chapelets d'insultes regorgent de ces vocatifs ou marqueurs de ciblage, le plus fréquent (et souvent le plus insultant) étant «thou».

Injurier, c'est en effet s'exclamer. Les commentaires métalinguistiques émis par certains personnages shakespeariens associent à plusieurs reprises l'injure à l'exclamation. Le Chief Justice, assistant à l'altercation entre Falstaff et l'hôtesse (Mistress Quickly) dans 2 Henry $I V$, réagit comme suit : 
Fie! what man of good temper would endure this tempest of exclamation?

Par le «Fie» ${ }^{21}$ qui introduit sa remontrance, l'homme de loi s'inscrit ironiquement dans la logique de la rhétorique exclamative qu'il dénonce, ce «fie» traduisant l'aspect contagieux de l'insulte et de la violence verbale en général, qui finit parfois par gangrener les membres de l'assistance et contribue à une inflation du volume sonore. A l'époque élisabéthaine les termes «exclaim» et «exclamation» signifient d'ailleurs le plus souvent «accuse loudly», «reproach», terme dont il est inutile de rappeler qu'il est beaucoup plus fort que le mot «reproche» français et qu'il veut souvent dire «insulte» 22 . Il n'est pas surprenant que les termes «exclamation» ou «exclaim» soient présents dans Richard $I I I$, pièce qui regorge de malédictions et de violences verbales de toutes sortes. A en croire Anne, le démon Richard a, par sa vilenie, fait naître sur terre un «bruit d'enfer» («For thou hast made thy happy earth thy hell») fait de malédictions et d'imprécations («Fill'd it with cursing cries and deep exclaims» ${ }^{23}$ ). Dans Richard III, la voix des femmes devient incantatoire, l'injure rejoignant ainsi la prophétie ${ }^{24}$. L'exclamation est en effet le mode d'expression favori des personnages féminins dans cette pièce, comme le confirme le discours de la Duchesse d'York lorsque, mettant en œuvre une véritable pédagogie de l'insulte, elle donne à Élisabeth le conseil suivant : «Be copious in exclaims» (IV.4.132-5). Les termes «exclamations» et «insult» finissent par se confondre dans la réaction de Richard aux agressions verbales des deux femmes: «Thus will I drown your exclamations» (IV.4.144-54) dit-il, mettant en concurrence le bruit de ses trompettes et de ses tambours («flourish and alarums») avec le bruit de l'injure, accentuant ainsi la dimension sonore de l'insulte et réduisant la voix des deux femmes au statut de bruit importun. Notons que l'on peut lire dans cette confrontation, l'évocation ironique de la réalité acoustique de la représentation, la voix des jeunes acteurs incarnant les rôles féminins ne pouvant rivaliser avec les clameurs des voix adultes ou des trompettes.

La nature exclamative de l'insulte, mise en évidence dans ces commentaires, a des implications sonores. S'exclamer signifie en effet s'écrier («ex-clamare»), ce qui suppose que l'insulte en général ne se dit pas mais se crie, et que le «dire» prévaut sur le «dit». L'injure est donc cri de guerre dont la vocalisation semble être le prolongement naturel d'un certain type de syntaxe ${ }^{25}$. 
Cependant, il suffit de lire et de mettre en voix quelques bordées d'injures pour se rendre compte que le volume sonore ne suffit pas à épuiser la vocalisation de l'injure shakespearienne. La dernière production de Troilus and Cressida à laquelle j'ai assisté au Swan ${ }^{26}$ était de ce point de vue très révélatrice. La voix de l'injure y était tantôt amplifiée par l'utilisation d'un micro (ironiquement celui-là même qui avait servi à mettre en voix le discours d'Ulysse), tantôt neutralisée lorsque Thersite et Patrocle, côte à côte, s'injuriaient calmement en ces termes :

Pat. Why thou damnable box of envy, thou, what meanst thou to curse thus?

Ther. Do I curse thee ?

Pat. Why, no, you ruinous butt, you whoreson indistinguishable cur, no.

Ther. No? Why art thou then exasperate, thou idle immaterial skein of sleave-silk, thou green sarcenet flap for a sore eye, thou tassel of a prodigal's purse, thou

(TRO, V.1.24-31)

Dans cet extrait, l'insulte est, à chaque fois qu'elle apparaît, précédée d'un vocatif, qu'il s'agisse de «you» ou de «thou», qui montre que l'on ne va pas tarder à tirer ${ }^{27}$, qui peut inviter à élever la voix mais qui peut aussi constituer un temps mort, permettant à l'insulteur de reprendre son souffle ou de retrouver à la fois sa respiration et son inspiration. «Breathe awhile and then to it again» dit Hal à Falstaff qui n'en finit pas de l'insulter dans 1 Henry IV (II.4.241). La syntaxe exclamative et énumérative de l'injure illustrée dans cette pièce ${ }^{28}$ mais aussi dans les chapelets cités plus haut réclame une gymnastique de la voix et du souffle. Pour se répandre en «foul word» et en «foul wind» $(A D O$, V.2.52-3), encore faut-il avoir $\mathrm{du}$ souffle et savoir le contrôler. L'injure Shakespearienne, lorsqu'elle est intégrée à un chapelet se caractérise également par son rythme, rythme rendu par la voix et qui repose sur une rhétorique accumulative mais aussi souvent fondée sur la gradation qui peut trouver son expression vocale, dans une augmentation du volume. On peut en effet remarquer que l'énumération d'injures produit une musique, reposant sur des rythmes binaires, ternaires, saccadés ou plus souples. Une accumulation de monosyllabiques telle que «thou drone, thou snail, thou slug, thou sot» (ERR, II.2.194) produit un effet musical. Les diphtongues y précèdent les voyelles brèves, produisant 
une accélération du rythme, doublé d'une allitération dont l'effet est lui aussi musical. S'il est bien difficile de dégager ce que seraient «les sons privilégiés» de l'insulte, on peut néanmoins constater que certains chapelets d'injures reposent sur une répétition de sons, qu'il s'agisse d'allitérations ou d'assonances, répétitions qui s'entendent d'autant mieux qu'elles sont très souvent anaphoriques. Il suffit, pour s'en convaincre, de citer quelques séries d'injures telles que : "With silken, sly, insinuating Jacks» (RIII, I.3.53), «Thou wretched, rash, intruding fool» (HAM, III.4.32), «Here, thou incestuous, murd'rous damnèd Dane» (HAM, V.2 278), «Thou say, thou serge, nay, thou buckram lord!» (2HVI, IV.7.23), "What an errant, rascally, beggarly, lousy knave it is» ( $H V$, IV.8.356), ou enfin «That little little, less than little wit» (TRO, II.3.11-2) qui résonne comme un «tongue-twister» et qui semble renouer, par sa dimension allitérative, avec ce que Jacques VI appelle «Tumbling verse», forme de vers dont il recommande l'usage pour les invectives et les «flytings» ${ }^{29}$. Enfin, les effets d'échos très souvent produits par l'usage de stichomythies lors des combats d'insultes tendent eux aussi à faire ressortir une musique de l'injure.

Ainsi, les discours théoriques et métalinguistiques sur l'injure et sa voix que nous avons abordés en premier lieu et qui faisaient de cette voix un bruit semble être contredits par la pratique shakespearienne de l'injure qui en fait presque une musique. Entre bruit et musique, l'injure devient cette «discorde musicale» dont parlent Hippolyta et Thésée dans A Midsummer Night's Dream (IV.1.102), pièce où le «flyting» (combat d'injures) est vu par Puck comme un spectacle musical («jangling», $M N D$, m.2.353), une musique discordante qui ne peut manquer de rappeler les manifestations populaires et carnavalesques élisabéthaines.

II semble donc qu'avec l'insulte, la voix prévaut sur le sens, que les paroles s'effacent derrière le bruit ou la musique, que le dit s'évanouit derrière le «dire» et le faire, que le signifié s'estompe derrière le signifiant. Mais lorsque l'on examine les insultes shakespeariennes de façon globale, il ressort que l'énumération et l'accumulation répondent à une logique de sens autant qu'à une logique formelle, c'est-à-dire musicale. Même si l'on doit parfois entendre la musique de l'injure, le mécanisme qui préside à la formation d'un chapelet est également sémantique. Le thème musical ne peut faire oublier le thème lexical et métaphorique mis en voix par l'insulteur qui s'efforce d'épuiser un «sujet». Dans The Taming of the Shrew, le chapelet d'injures que Petruchio dévide au petit tailleur répond à une logique de cohérence sémantique. Il 
s'emploie à explorer par l'injure le domaine de la couture tout en jouant sur la polysémie de mots tels que «yard» :

Thou liest, thou thread, thou thimble, thou yard, three-quarters, half-yard, quarter, nail (SHR, IV.3.107-8).

Away, thou rag, thou quantity, thou remnant ( $S H R$, IV.3.111).

Dans The Merry Wives of Windsor, les injures que Ford lance à celui qu'il prend pour une sorcière se suivent et se ressemblent sémantiquement: "Out of my door, you witch, you rag, you baggage, you polecat, you runnion, out, out !» (WIV, IV.2.170-1). Même si l'on peut entendre ce chapelet avec l'oreille du musicien, il ne faut pas négliger d'en noter la cohérence sémantique. Cela est flagrant dans 1 Henry $I V$ où les chapelets de Hal et de Falstaff sont de véritables variations sur un thème donné : la querelle des gros et des maigres. Cependant, les redondances qui caractérisent les chapelets qui viennent d'être évoqués, laissent à penser que le dire est au moins aussi important que le dit, que l'énonciateur prend plaisir à écouter sa voix, à s'entendre débiter des injures dont il déguste le son peut-être plus que le sens. Le spectateur est alors tiraillé entre le plaisir d'entendre et l'envie de comprendre. Or le dramaturge manipule ces deux facettes en explorant divers modes de vocalisation de l'injure et en travaillant les rapports entre la voix et le message, le dit et le dire.

Chez Shakespeare toutes les injures ne sont pas dites d'une même voix, ni sur le même ton. L'injure peut au contraire se dire sur tous les tons. Injures littéraires et injures de théâtre, les insultes shakespeariennes et leur manifestation vocale s'intègrent à des situations dramatiques précises et diverses et peuvent varier en fonction notamment des déplacements dans l'espace des acteurs. L'acte de parole qui consiste à injurier semble bénéficier d'un traitement spécial quant aux rapports entre l'espace et la voix. La logique qui voudrait que le volume vocal soit proportionnel à la distance entre deux interlocuteurs, la logique qui voudrait que plus on se trouve loin de l'interlocuteur, plus on parle fort, ne semble pas être respectée lorsqu'il s'agit d'injure. Edward T. Hall, le père de la proxémique le note, qui écrit : 
Des individus très en colère ou très désireux de convaincre leur interlocuteur se rapprocheront de celui-ci et tourneront en quelque sorte «le bouton de l'intensité» en criant. ${ }^{30}$

La voix et la distance se désolidarisent, ce qui aboutit à une sorte de redondance de la violence qui s'exprime alors à la fois par la voix et par le déplacement dans l'espace. Le volume de la voix augmente d'autant plus que l'on se rapproche de sa cible. Mais on peut tout à fait concevoir que le personnage du couard dise ses injures à mi-voix et en plus de loin, ce qui contredirait la relation logique (plus on est loin, plus on parle fort). Une injure peut se susurrer ou se hurler, se «rire» ou se «pleurer», se dire d'une voix câline ou tonitruante. Cependant les variations vocales de l'injure sont parfois imposées par le texte shakespearien et les situations dramatiques qu'il évoque. L'analyse de quelques exemples suffit à le montrer.

Dans 1 Henry $I V$, la dimension spectaculaire du combat d'injures entre Hal et Falstaff impose que la voix soit projetée afin qu'elle atteigne non seulement l'adversaire mais aussi le public de la taverne, présent sur scène. L'injure en aparté au contraire impose à l'acteur de retenir sa voix tout en faisant en sorte qu'elle soit audible pour le public. Lorsque l'injure s'intègre à cette $\cdot$ convention théâtrale, une tension peut naître alors entre une rhétorique du cri qui devrait trouver son prolongement sonore et une voix qui doit être retenue. La voix de l'aparté est une voix de théâtre par excellence, audible pour le public, elle ne doit pas l'être pour les personnages ou en tout cas, le public doit croire qu'elle ne l'est pas. La voix de l'aparté est par essence une double voix, une voix que l'on entend de deux oreilles.

Dans Much Ado About Nothing, il faut, comme c'est le cas dans la version de la $\mathrm{BBC}$, que Benedick dise son injure à Claudio («you are a villain» ${ }^{31}$ ) sur le ton de la confidence et du secret. II le prend en effet à part et les répliques qui suivent son défi à Claudio montrent que Don Pedro n'est pas censé entendre ce qui se dit entre les deux hommes. Cette discrétion de l'injure respecte l'esprit des codes de duel que Shakespeare réécrit assez fidèlement ici. Le duelliste doit se contrôler, «dompter» ses paroles et sa voix. S'il lance une injure, celle-ci doit être une accusation. Il n'est pas digne d'un homme d'honneur d'élever la voix car cela serait le signe d'une 
perte de contrôle de soi, d'une domination du corps sur l'esprit. Dans ce cas, la voix s'efface derrière le sens, le dit prévaut sur le dire. A la Renaissance, les manuels consacrés au duel ${ }^{32}$ prônent cet effacement de la voix et du corps et imposent des formules et des codes qui étouffent la voix personnelle de l'énonciateur, étouffement qui est rendu dans cette scène par l'utilisation de l'aparté. L'effacement de la voix et du corps prôné par les maîtres du duel trouve alors son pendant dramaturgique dans le recours à l'aparté. En utilisant la formule toute faite «you are a villain», Benedick met sa voix «à part» («aside») physiquement et métaphoriquement. L'utilisation des cartels, c'est-à-dire de l'injure écrite et contrôlée, permettait cet effacement de la voix. Dans Twelfth Night (III.4), Shakespeare transgresse ce code des duels en donnant voix au cartel concocté par Sir Andrew. Lorsqu'il lit tout haut, et d'une voix que l'on imagine tonitruante, ce qui ne devait être qu'un message écrit, Sir Toby redonne corps à une injure qui était censée être sans voix ( «I will deliver his challenge by word of mouth», III.4.184). Cette transgression est à l'image d'une lettre qui bafoue tous les codes relatifs aux cartels et qui laisse présager l'avortement du duel annoncé. Shakespeare exploite donc différents modes de vocalisation. Une injure discrète n'a pas le même effet sur le spectateur qu'une injure tonnante et donne des personnages et de l'injure des images différentes.

Shakespeare sait enfin jouer, à des fins comiques, sur le décalage entre le ton et le contenu, le son et le sens du discours. $C$ 'est le cas dans Henry $V$ où Fluellen insulte et humilie Pistol sur un ton des plus courtois, lui disant tour à tour «God pless you Ensign Pistol, you scurvy lousy knave, God pless you» (V.1.15-6), «I peseech you heartily, scurvy lousy knave» (V.1.20), ou encore «Will you be so good, scald knave, as eat it ?» (V.1.26-7). Le mélange d'adresses polies et d'injures impose à l'acteur d'insulter courtoisement, avec ce que ce ton paradoxal peut avoir de comique ${ }^{33}$. Dans 2 Henry $I V$, les injures lancées par Doll Tearsheet illustrent également ce décalage entre le signifiant et le signifié, puisqu'elle câline le gros Falstaff à coups de «villain» («You whoreson little valiant villain», II.4.206) et de «rogue» («You sweet little rogue», II.4.213), transformant la voix de l'injure en caresse. Dans $A$ Midsummer Night's Dream, le prologue de Quince relève de ce «grand écart» entre le dit et le dire. La voix vient contredire le sens. Emis comme un compliment, le discours de l'artisan se transforme en injure par un défaut de vocalisation. Mal ponctué, le prologue annonce les faiblesses de toute une pièce (Pyramus and Thisbe) jouée par des 
artisans qui, malgré tous leurs efforts, ne savent pas mettre en voix, contrairement à ce ventriloque de Puck qui peut, lui, prendre toutes les voix. Les formules oxymoriques prononcées par Bottom au début de la pièce, telles que «[...] I will roar you as gently as any sucking dove. I will roar you an 'twere any nightingale» (I.2.74-5) ou encore «I'll speak in a monstrous little voice» (I.2.48), prennent alors tout leur sens et deviennent emblématiques des fausses notes qui caractérisent le spectacle de Pyramus et Thisbe.

II est donc mille et une façons de vocaliser l'injure, ce qui n'est pas étonnant puisque l'on parle d'un temps où l'insulte était même mise en chant. "An I have not ballads made on you all and sung to filthy tunes, let a cup of sack be my poison» dit Falstaff, qui, animé par un esprit de vengeance dans 1 Henry IV (II.2.42-3), évoque ainsi l'image paradoxale d'une insulte chantée. Ce chant d'insultes que constitue la ballade effraie Cléopâtre, qui, certaine de devenir la victime de rimailleurs, prédit à la fin d'Antony and Cleopatra:

Saucy lictors

Will catch at us like strumpets, and scald rhymers Ballad us out o'tune. [...]

(V.2.214-21)

Insulter et chanter, ces deux actes qui semblent a priori irréconciliables sont réconciliés à l'époque élisabéthaine, tout comme ils le sont dans Twelfth Night où Sir Andrew se propose d'entonner un singulier canon lorsqu'il lance à ses compères «Let our catch be "thou knave"» (TN, II.3.68-9). Cet étrange mariage vocal fait partie intégrante d'un univers carnavalesque et du «double ton» ${ }^{34}$ qui le caractérise.

Ainsi, entre le dit et le dire, entre le dire et le faire, l'injure devient emblématique du théâtre et d'un art dramatique où «l'action est éloquence» et où les mots prennent corps. Lieu de vocifération, l'injure est à l'image du théâtre où il importe de projeter une voix qui agit même si elle n'est que «vent». Cette prédominance de la voix au théâtre apparaît clairement lorsque l'on se rappelle que les Élisabéthains allaient non pas "voir» une pièce mais l'entendre («hear a play»). Excès de langage, I'injure prête aux excès de voix. Cependant, la pratique shakespearienne de l'injure ne se résume pas à cette seule équation. Certes les insulteurs se font souvent «hauts-parleurs», mais Shakespeare sait aussi exploiter toutes les ressources dramatiques offertes par cet acte de parole, en étouffant, 
amplifiant ou décalant sa voix. C'est cette voix et ses variations qui peuvent faire de l'injure tantôt une arme, tantôt un instrument de séduction, tantôt un bruit, tantôt une musique, tantôt une violence, tantôt une caresse et parfois tout cela en même temps.

Nathalie VIENNE-GUER RIN

C.E.T.A.S.

Université de Rouen

\section{NOT E S}

${ }^{1}$ Dans Henry $V$, Pistol, interprétant le terme «solus» comme une injure, lance à Nim «I do retort the solus in thy bowels» (HV, II.1.42).

2 «She speaks poniards and every word stabs» (ADO, I.1.246-7).

${ }^{3}$ Les gros mots, Que sais-je ?, P.U.F., 1975, p. 27-40.

${ }_{5}^{4}$ Ibid., p. 34.

${ }^{5}$ Ibid., p. 34-5.

${ }^{6}$ Ibid., p. 35.

${ }^{7}$ Évelyne Largueche, Injure et sexualité, P.U.F., 1997, p. 95.

${ }^{8}$ Ibid., p. 95.

${ }^{9}$ Ibid., p. 78.

${ }^{10}$ The Arte of English Poesie (1589), Menston, The Scholar Press Limited, 1968, p. 46.

${ }^{11}$ Évelyne Largueche, op. cit., p. 95-6.

12 ADO, II.1.246-7.

${ }^{13}$ HAM, III.2.379.

${ }^{14}$ Dans L'effet injure (P.U.F., 1983, p. 109), Évelyne Largueche définit ce qu'elle appelle «l'injure non-spécifique» comme suit : «Nous dirons qu'il y a injure non-spécifique lorsque l'injurieur établit son injure (donc au niveau des procédés), à partir des mots utilisés non plus comme des porteurs de sens, mais comme substituts et dont le pouvoir tient précisément à ce qu'ils sont mis à la place de ce qu'ils sont censés représenter».

${ }^{15}$ Évelyne Largueche, Injure et sexualité, op. cit., p. 136.

${ }^{16}$ Ibid., p. 138.

17 Ibid., p. 143. L'auteur évoque les joutes ritualisées telles qu'elles sont décrites par William Labov dans Le parler ordinaire, Paris, Éd. de Minuit, 
1978. Voir William Labov, Language in the Inner City, Oxford, Basil Blackwell, 1977 (1972).

${ }^{18}$ Le chapitre se termine comme suit : «A small trembling voyce proceedeth from feare, and such an one commonly have great Oratours, or at least, it were good they should have, in the beginning of their Orations, for thereby they winne a certain compassion and loving affection of their auditors. Much more might be said of this subject ; but for that it concerneth specially physiognomy, \& natural constitution of the organs and humours of the bodie, therefore I will omit it» (Thomas Wright, The Passions of the Mind (1601), Hildesheim, New-York, Georg Olms, Verlag, 1973, p. 211-2).

19 A ce sujet, voir David Bevington, Action is Eloquence, Harvard U. P., 1984, chap. III.

${ }^{20}$ Le terme «thunder» revient assez souvent pour décrire l'invective dans sa dimension sonore. Winchester s'exprime en ces termes dans IHVI: «And that engenders thunder in his breast / And makes him roar these accusations forth» (II.1.39-40). La pucelle emploie le même terme un peu plus loin : «If Talbot do but thunder, rain will follow» (1HVI, III.2.59). Queen Gertrude réagit à la violence verbale d'Hamlet par cette question: «What have I done, that thou dar'st wag thy tongue/In noise so rude against me ?» (HAM, III.4.38-9). Aaron, surprenant Chiron et Demetrius en plein combat verbal, leur conseille de surmonter cette discorde : «And join for that you jar» (TIT, II.1.103). Le terme «jar» revient très souvent dans $1 H V I$, ce qui n'a rien d'étonnant étant donné que cette pièce n'est que discorde. Ainsi, Bedford l'emploie tout d'àbord : «Cease, cease these jars and rest your minds in peace» (I.1.43). King Henry quelques scènes plus loin utilise aussi ce terme : «That two such noble peers as you should jar !» (III.1.69). Exeter parle de «this jarring discord of nobility» (IHVI, IV.1.188) Dans $2 H V I$, le roi utilise à nouveau cette image : «The winds grow high, so do your stomachs, lords / How irksome is this music to my heart ! / When such strings jar, what hope of harmony? / I pray, my lords, let me compound this strife» (2HVI, II.1.56-9).

${ }^{21}$ Au sujet des interjections, voir Richard Carew, The Excellency of the English Tongue (1595-6): "Again, for expressing our passions, our interjections are very apt and forcible : [...] In detestation wee saye Phy, as if there withall we should spitt ; [...]», in Elizabethan Critical Essays, ed. Gregory Smith, Oxford U.P., 1904, (1964), Volume II, p. 287.

${ }^{2}$ R. A. Foakes, dans l'édition Arden de HVIII, note que le terme «exclamation» est toujours utilisé par Shakespeare dans le sens de «reproach», ce qui confirme ce lien essentiel entre l'insulte et l'exclamation. Pour le terme «reproach», voir OED : «Sens 3. Blame or censure directed against a person, sometimes implying abusive or opprobrious language, but also (esp. in mod. use) applied to mild upbraiding or rebuke. Sens 4. a. An insult in act or deed. b. An expression of disapproval, censure, reproof, or upbraiding; a verbal insult, an opprobrious expression or epithet». 
${ }^{23}$ RIII, I.2.50-2.

${ }^{24}$ Voir Lisa Jardine, Still Harping on Daughters, Sussex, Harvester Press, 1983, p. 115-8. Voir «Grey. Now Margaret's curse is fallen upon our heads, / When she exclaim'd on Hastings, you and I, / For standing by when Richard stabb'd her son» (RIII, III.3.15-7).

${ }^{25}$ Sur le lien entre rhétorique et voix, voir Keir Elam, «Inelocutio. Shakespeare and the rhetoric of the passions», in Shakespeare, Rhétoriques du texte et du spectacle, Les Belles Lettres, 1992, p. 143-56.

${ }^{26}$ Stratford, décembre 1998, mis en scène de Michael Boyd.

27 Il faut noter que Patrocle emploie tantôt le «you» tantôt le «thou» pour s'adresser à Thersite, ce qui tend à prouver que les deux modes d'adresse se confondent ici.

${ }^{28}$ A ce sujet, voir Nathalie Vienne-Guerrin, «L'anatomie de l'insulte dans 1 Henry IV», Bulletin de la société de stylistique anglaise, $\mathrm{n}^{\circ} 17,1996$.

${ }^{29}$ A Short Treatise on Verse : «Let all your verse be Literall, so far as may be, quatsumeuer kynde they be of, bot speciallie Tumbling verse lor flyting. Be Literall I meane that the maist pairs of your lyne sall rynne upon a letter, as this tumbling lyne rynnis vpon $\mathrm{F}$.

Fetching fude for to feid it fast furth of the Farie.

Ye may obserue that thir Tumbling verse flowis not on that fassoun as vtheris dois. For all vtheris keipis the reule quhilk I gaue before, to wit, the first fute short, the secound lang, and sa furth. Quair as thir hes twa short and ane lang throuch all the lyne, quhen they keip ordour : albeit the maist pairs of thame be out of ordour and keipis na kynde nor reule of Flowing, and for that cause are callit Tumbling verse : except the short lynis of aucht in the hinder end of the verse, the quhilt flowis as vther verses dois, as ye will find in the hinder end of this buke, quhair I giue exemple of sindrie kyndis of versis» (Elizabethan Critical Essays, ed. Gregory Smith, Oxford U. P., 1904 (1964), Volume I, p. 218-9).

30 La Dimension cachée, Seuil, 1971 (1966), p. 144.

31 ADO, V.1.143.

32 Voir notamment Vincentio Saviolo, His Practice. In two Books, London, 1595.

${ }^{33} \mathrm{Si}$ Shakespeare joue sur le décalage comique entre le ton et le contenu de l'injure, il sait aussi intensifier l'injure en l'intégrant à une atmosphère apocalyptique. Dans King Lear, les malédictions sont proférées dans un bruit de tempête. Le bruit de l'injure trouve alors son pendant naturel dans le vacarme du tonnerre.

${ }^{34}$ Nous empruntons l'expression à Mikhaïl Bakhtine, L'œuvre de François Rabelais et la Culture populaire au Moyen Age et à la Renaissance, Paris, Gallimard, 1970, p. 25, 167, 248-9, 407, 412-17, 454. 